\title{
AdAPTIVE RANDOM SPATIAL BASED CHANNEL ESTIMATION (ARSCE) FOR MILLIMETER WAVE MIMO SYSTEM
}

\author{
Rajkumar M Vadgave ${ }^{1}$, Manjula $\mathrm{S}^{2}$ and T S Vishwanath ${ }^{3}$ \\ ${ }^{1}$ Department of Electronics \& Communication Engineering, \\ Veerappa Nisty Engineering College, Shorapur, India \\ ${ }^{2}$ Department of Electronics \& Communication Engineering, \\ Lingaraj Appa Engineering College, Bidar, India \\ ${ }^{3}$ Department of Electronics \& Communication Engineering, \\ Bheemanna Khandre Institute of Technology, Bhalki, India
}

\begin{abstract}
Millimeter-wave and mMIMO communications are the most essential success systems for next-generation wireless sensor networks to have enormous amounts of accessible throughput and spectrum. Through installing huge antenna arrays at the base station and performing coherent transceiver processing, IMIMO is a potential technology for enhancing the bandwidth efficiency of wireless sensor networks. The use of mmWave frequencies for mMIMO systems solves the problem of high path-loss through offering greater antenna gains. In this work, we provide a design with a random spatial sample structure that incorporates a totally random step before the analogue is received. It contains a totally random step before the analogue received signals are sent into the digital component of the HBF receiver. Adaptive random spatial based channel estimation (ARSCE) is proposed for channel session measurement collection, and an analogue combiner with valves has been used to estimate the signals at each receiving antenna. The proposed optimization problem formulation attempts to discover the orientations and gains of wideband channel routes. In addition, our proposed model has compared to various state-of-art techniques while considering error minimization.
\end{abstract}

\section{KEYWORDS}

Millimeter-wave (mmWave), massive multi-input-multi-output (mMIMO), Hybrid Beamforming (HBF), Channel Estimation, Error Minimization.

\section{INTRODUCTION}

Modern broadband cellular systems will have to keep up with the demand for high-speed data capabilities, which is expected to skyrocket. Two major ways to meet these needs are largeantenna-array multiple-input-multiple-output (MIMO) designs (also known as massive MIMO / m-MIMO) and millimeter wave (mmWave) systems. The mMIMO is preferred when the objective is to enhance spectral and energy efficiency, whereas the mmWave approach maximises a broader bandwidth [1]. Many contemporary commercial mobile network systems operate at frequency band below $6 \mathrm{GHz}$, whereas millimeter wave communications utilise the spectrum from roughly $30 \mathrm{GHz}$ to $300 \mathrm{GHz}$. The major benefit of owning at higher frequencies is the possibility of significantly greater bandwidth channels [2]. 
The mmWave communications potential for cellular networks has been acknowledged in [3], as well as its ability to offer extremely high throughput. Even in non-line-of-sight conditions, [4] [5] claimed a transmission distance of far more than $200 \mathrm{~m}$. For 5th generation (5G) cellular networks and beyond, millimeter wave communications are of interest [6]. Modern signal processing approach are required to make mmWave communication systems commercially viable [4], [6]. There are several reasons behind this: new hardware restraints develop as a result of operating at larger bandwidths and higher frequencies and, mmWave channel characteristics change. Significant antenna arrays are required to achieve large beamforming gains, which brings additional power consumption and circuit implementation issues.

Conventional digital precoding is too expensive to implement in millimeter-wave communication systems. As a result, hybrid precoding is a superior option that works at mixes of digital and analogue precoding. It's generally based on one of two types of structures: subconnected and completely linked, whereas the completely linked structure has been intensively explored in the academic world because it can reach the theoretical ideal spectrum efficiency. In [4], the spatial sparsity of the mmWave channel was used to reconstruct the hybrid precoding problem. The orthogonal matching pursuit (OMP) method was suggested to tackle the problem and greedy algorithm is presented based on the OMP method in [7].

The antenna array shape is not taken into account by the method, which substantially decreases computing complexity while maintaining high performance. By choosing an appropriate threshold, an orthogonal matching pursuit method with performance similar to the optimum and higher than the OMP algorithm is provided [5]. Even though OMP approach has good speed, it has a rather high level of complexity, so as a result, [6] investigates a high-performance and realtime precoding approach based on SVD (singular value decomposition). Overall performance of this technique is comparable to the OMP algorithm, but the complexity is significantly lower. An alternative minimization method based on the concept of manifold optimization is proposed in [8], which reduces the program's complexity. Many researchers have been drawn to the subconnected structure because of its features, which allow it to strike a proper balance between cost and performance. However, unlike fully connected structure, which has a one-to-one correlation between both the antenna and the RF chain, each RF chain in the subconnected configuration is only linked to a portion of the antenna. As a result, in recent years, hybrid precoding techniques using subconnection architectures has gotten a lot of interest.

With the aid of semidefinite relaxation, an alternative minimization method for the subconnected structure is also constructed in [8]. In a similar vein, [9] proposes a novel divide-and-conquer precoding method, this scheme's performance is comparable to SDR-alternative minimization, that can save time and is resistant to potential saddle spots with underperformance. In [10], a hybrid precoding method based on SIC (successive interference cancellation) is presented in response to the hybrid precoding challenge of energy-saving subconnection design. It eliminates the need for matrix inversion and SVD and it has a considerably low computational cost than standard sparse reconstruction precoding techniques. The sparsity and low rank characteristics of specific wireless channels were recently combined for effective channel state information (CSI) estimation in [11] [13]. In [12], a two-step approach (one stage per channel attribute) was developed for narrowband mmWave MIMO channel estimation with Hybrid Beamforming (HBF) transceivers. In [13] proposed a matrix completion method that took advantage of these features for the same transceivers and narrowband channels with simple switches. This approach outperformed the approach of [12] in terms of estimate performance while needing just brief channel sounding periods. In [14], wideband mmWave MIMO channels featuring frequency selectivity were recently introduced, which offered a CSI estimation approach that used the sparsity of the channel in frequency and time domains. 
In this paper, we provide a unique analogue combining design with a random spatial sampling layout, which includes a purely random stage before the analogue received signals are fed into the HBF receiver's digital component. This design is utilized for channel session measurement gathering, and an analogue combiner with valves was used to approximate the signals at receiving antennas individually. The proposed model named as adaptive random spatial based channel estimation (ARSCE) and this design major feature is the random choice of available analogue receive beams during channel period. It combined with the proposed CSI estimation approach, results in enhanced performance with short training periods. The matrix generated at the HBF receiver following analogue processing of training signals containing multiple receiving beams has the same rank as the wideband mmWave m-MIMO channel matrix. Based on this discovery, we devise a CSI estimate optimization model that takes use of both the low-rank feature of the sparsity of the channel matrix and the received training signal matrix in the beamspace space. The suggested optimization problem formulation seeks to identify the wideband channel routes' orientations and gains. The distinctions are in the mathematical formulas. The low-rank feature of narrowband mmWave m-MIMO channels is used to determine the measurement needs for them and the scalability of the technique in wideband mmWave mMIMO channels.

\section{LiTERATURE SURVEY}

In m-MIMO systems, hybrid beamforming designs are developed to reduce hardware and training overhead costs. The carrier frequency (mmWave), CSI (average or instantaneous), and complexity of hybrid beamforming may all be categorized to reduce the switched complexity. The method to use to achieve the optimal tradeoff between these factors is determined on the application and channel characteristics [15], while obtaining specific and reliable CSI for Multi user-mMIMO systems at millimeter waves is difficult because to the large number of BS antennas. In mMIMO, a combined iterative approach based on step-length optimization may be used to obtain accurate channel estimate [16]. In the context of faulty CSI and hardware restrictions, a beamforming neural network (deep learning networks) for mmWavemMIMO systems can improve the beamforming layout and is resilient with greater spectrum efficiency as compared to standard beamforming methods [17]. In mMIMO systems, the ideal number of UEs programmed concurrently for a specific number of BS antenna components offers maximum spectrum efficiency. The frequency spectrum of downlink (DL) and uplink (UL) can be the same (allowing combined network improvement), and it is also independent of UE locations at any given time [18]. In comparison to existing methods, the multitask deep learning (MTDL)-based HBF algorithm for mmWavemMIMO OFDM systems can provide good results in terms of runtime and sum-rate [19].

Based on the arrival/departure angle and with the fewest number of pilots, an effective channel estimate technique for the time-varying DL channel of mmWave MIMO systems is suggested [20]. With flawless CSI and a low-complex single-cell DL mMIMO HBF system, the sum-rate approaches optimal network throughput is shown in [21]. The performance difference between hybrid and digital beamforming can be narrowed for a specific number of Relay nodes by decrease the quantity of multiplexed symbols [22]. In comparison to OMA systems, clustering and feedback-based hybrid beamforming for DL mmWave MIMO NOMA systems provide highest sum-rates [23]. A multi user (MU) detection technique based on a "markov random field" for model cluster sparsity and predict mMIMO channel outperforms systems which do not take use of channel cluster sparsity [24]. In developing hybrid beamforming for broadband mmWave MIMO systems, eigenvalue decomposition, manifold optimization, and OMP algorithms give BER and spectral efficiency (SE) that is closer to completely digital BF solutions [25]. When the number of RF chains in a mmWavemMIMO system increases, the best hybrid 
combining and precoding approaches utilising the OMP algorithm to achieve total sum-rates that are comparable to completely digital precoding [26].

Hybrid beamforming with user scheduling technique is described for DL to increase throughput (near to completely digital beamforming) of mmWavemMIMO OFDM systems (users with similar strongest beams). Digital beamforming is used to achieve the highest performance increase (by minimising excess inter-user congestion) [27] and analogue beamforming vectors are utilised to determine the ideal beam of each consumer. For mmWave MIMO uplink channels, a low computational complexity hybrid beamforming system minimises inter-user interference while performing similarly to a completely digital beamforming architecture. In mmWavemMIMO systems based on Generalized Low Rank Approximation of Matrices, the coordinated RF beamforming method requires just composite CSI rather than the entire physical channel matrix.

In both FDD and TDD systems, this method gives a competitive option by addressing the coordination between BS and UEs to get maximum array gain with no dimensionality limitation [22]. HBF is used in mmWavemMIMO relay systems to improve sum-rates via lowering the sum MSE between both the received signal of hybrid and digital BF schemes. The overall sum-rates rise as the correctness of arrival/departure angles and the number of RF chains rise [28] and the possibility of low-cost analogue optimal unconstrained precoder, RF hardware implementations, and combiner methods for mmWavemMIMO systems are proposed. The suggested methods' numerical findings demonstrated that the SE of mmWave systems with transceiver hardware restrictions approaches the unrestrained performance limits [29]. In mmWavemMIMO systems, low-complexity phased-ZF hybrid precoding is utilised in the RF domain to get substantial power gains and low-dimensional ZF precoding has been used in the baseband domain allowing multistream operations [30].

\section{Proposed Method}

A point-to-point $A_{R} \times A_{\text {Tlarge }}$ MIMO communication network functioning across wideband mmWave channels is considered, where transmitter is having $A_{\text {Tantennas and the receiver is }}$

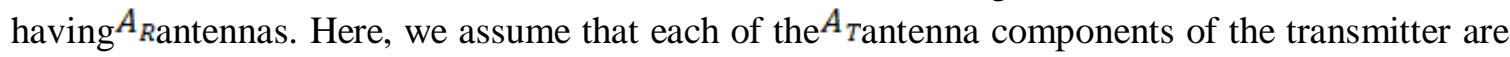
linked to a separate RF chain, whereas the receiver antenna elements are associated to $A_{R}$ that is less then $N_{R}$ at RF chains. Because of this hardware design, the transmitter may digitally precode up to $A_{T}$ separate signals from a single devoted RF link. We assume, on the other hand, that receiver is prepared with any of the known HBF designs [31] that enable both digital and analogue compounding. The considered mmWave MIMO network comprising wireless communication link of information $b_{c} \leq \min \left(A_{T}, A_{R}\right)$ that will be separate from data streams.

Initially, proposed the solution for large number of antennas by analog based HBF receiver at wideband MIMO channel. The assumption has been made where the communication system remains consistent throughout each frame but may change independently by one frame to the next. Each frame is made up of ${ }^{F}$ blocks allocated to channel estimates, with the rest of the frames allocated to data transmission. A greater $F$, of course, improves channel prediction while

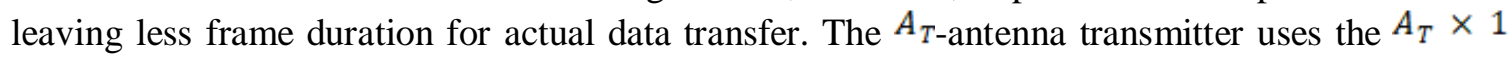
training symbols' vector $c[f]$ with each block $f$ with $f=1,2, \ldots, F$ to approximate the desired wideband mmWave MIMO channel. Ignoring the influence of Additive White Gaussian Noise (AWGN) for the sake of clarity, the $A_{R \text {-dimensional acquired training signal may be represented }}$ as: 
International Journal of Computer Networks \& Communications (IJCNC) Vol.14, No.1, January 2022

$$
\tilde{d}[f]=\sum_{e=0}^{E-1} G(e) c[f-e]
$$

This shows the convolution matrices of $E$ delay channel and training vectors of $E$ is $c[f-e] \in \mathbb{C}^{A_{T} \times 1}$. similar to eq. (1), is shown as;

$$
\tilde{d}[f]=\sum_{e=0}^{E-1} \sum_{h=0}^{A_{T}} g_{h}(e) c_{h}[f-e]
$$

where $g_{h}(e)$ indicates the $h_{\text {-th }}$ column of $G(r)$ and $c_{h}[f-e]$ the h-th element of $c[f]$. We may describe the inner convolution sum of the $E \times F$ Toeplitz matrix by altering the summation order in eq. (2). Specifically, by include the $E \times F$ Toeplitz matrix $h$, with its $(e, f)$-th element provided by:

$$
[\widetilde{D}]_{e, f}=c_{h}[f-E-e+2]
$$

Where, $\mathrm{h}=1,2, \ldots, A_{T}$ and $\mathrm{e}=0,1,2, \ldots, E-1$, and above eq. (2) can be written as

$$
\widetilde{D}=\sum_{h=1}^{A_{T}} \widetilde{G}_{h} \widetilde{\mathrm{M}}_{h}
$$

Where, $\widetilde{G}_{h}=\left[g_{h}(0) \ldots g_{h}(E-1)\right] \in \mathbb{C}^{A_{R} \times E}$ and $\tilde{Y} \in \mathbb{C}^{A_{R} \times F}$. The structure of $\widetilde{\mathrm{M}}$ and $\widetilde{G}$ are also reorganized to grouped at transmitting antennas and equation eq. (4) can be written as;

$$
\widetilde{D}=\widetilde{M} \tilde{G}
$$

Where, $\widetilde{\mathrm{M}}=\left[\mathrm{M}^{F}(0) \ldots \mathrm{M}^{F}(E-1)\right]^{F} \in \mathbb{C}^{E A_{T} \times F}$ and $\tilde{G}=[G(0) \ldots G(E-1)] \in \mathbb{C}^{A_{R} x E A_{T}}$. In addition, the decomposition of beamspace at every delay path matrix is;

$$
\bar{G}=N_{R} \bar{P}\left(I_{R} \otimes N_{T}^{G}\right)
$$

Where, $\bar{P}=[P(0) \ldots P(E-1)] \in \mathbb{C}^{A_{R} \times E A_{T}}$ and for the case of the wideband channel matrix $\bar{G}$, the decomposition of eq. (6) is identical to the beamspace decomposition of eq. (7). When all of the above information is combined, the matrix containing the received training symbols is provided by;

$$
\widetilde{D}=N_{R} \bar{P}\left(I_{R} \otimes N_{T}^{G}\right) \tilde{M}
$$

The difference between eq. (6) and eq. (7) is the rightmost matrix $\widetilde{\mathrm{M}}$, which includes the learning symbols. In our method, the latter equation will be utilised to represent the incoming training data in terms of the combined virtual channel gains $\bar{P}$. 
We provide the proposed channel estimation process issue description and comprehensive algorithmic solution. The sum of the low-rank matrix, which includes the learning signs following passing over the wideband mmWave MIMO link, and the AWGN matrix ${ }^{A}$ yields the received learning signal matrix at the outcome of the extended connector. In this paper, we using the low-rank feature of $\widetilde{D}$ to estimate the wideband channel matrix $\bar{P}$. To begin, the rank characteristics of the later matrix is considered for the sake of simplicity.

We design the following dual purpose optimization process for the estimate of $\bar{P}$, taking use of both the sparse composition of ${ }^{\bar{P}}$ and the low rank characteristic of the learning symbols' matrix $\widetilde{D}$.

$$
\min _{D, \bar{P}} Q_{R}\|D\|_{*}+Q_{p}\|\bar{P}\|_{1}
$$

Which subject to,

$$
\begin{gathered}
R_{\Omega}=\Omega(\mathrm{D}+\mathrm{A}), \\
D=U \bar{P} V
\end{gathered}
$$

Where D's fission norm in the optimization process that imposes its low rank characteristic, and

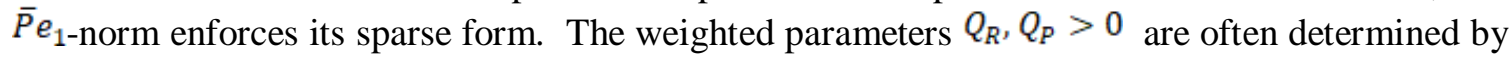
the number ${ }^{E}$ of unique mmWave MIMO channel propagation pathways. Because the noisy matrix $A$ is uncertain, we replace the first requirement in eq. (8) with its least-squares approximation $\left\|R_{\Omega}-\Omega * \mathrm{D}\right\|_{S}^{2}$ in the follows.

The ADMM method is used to solve the process eq. (8) optimally and the sampling is done on the channel matrix rather than the received learning signal, as indicated in the mathematical equation eq. (8). To solve eq. (8), we will do the following; restate the desired proces in the following equivalent form, we first incorporate the two input matrix variables $W=D-\mathrm{U} \bar{P} V$ and $Z \in \mathbb{C}^{A_{R} x F}$ :

$$
\begin{gathered}
\min _{D, \bar{P}, Z, W} Q_{R}\|D\|_{*}+Q_{P}\|\bar{P}\|_{1}+1 / 2\|W\|_{S}^{2}+1 / 2\left\|\Omega * Z-R_{\Omega}\right\|_{S}^{2} \\
\text { Subject to } D=Z \text { and } W=D-U \bar{P} V
\end{gathered}
$$

This is equal to eq. (8), except the cost function has now been dissected into four variables: $D, \bar{P}, W$ and $Z$. It is worth noting that the third component with in objective function now accounts for the discretization error, while the fourth term accounts for the AWGN interference noise. The optimization process of Lagrangian function readily written as:

$$
\begin{aligned}
& \mathcal{L}\left(D, \bar{P}, Z, W, X_{1}, X_{2}\right) \\
& \quad=Q_{R}\|D\|_{*}+Q_{P}\|\bar{P}\|_{1}+1 / 2\|W\|_{S}^{2}+1 / 2\left\|\Omega * \mathrm{Z}-R_{\Omega}\right\|_{S}^{2}+\operatorname{tr}\left(X_{1}^{G}(D-Z)\right) \\
& +\rho / 2\|D-Z\|_{S}^{2}+\operatorname{tr}\left(X_{2}^{G}(W-Z+U \bar{P} V)\right)+\rho / 2\|W-Z+U \bar{P} V\|_{S}^{2}
\end{aligned}
$$

where $X_{1} \in \mathbb{C}^{A_{R} x T}$ and $X_{2} \in \mathbb{C}^{A_{R} x 1}$ are dual factors (the Lagrange multipliers) that add the requirements of eq. (10) to the cost function, and ${ }^{\rho}$ indicates the ADMM stepsize. The following distinct sub-problems must be addressed at the $\mathrm{i}$-th algorithmic step, with $i=0,1, \ldots, I_{\max }$, as per the usual ADMM approach:

$$
X_{1}^{(i+1)}=X_{1}^{(i)}+\rho\left(Z^{(i+1)}-D^{(i+1)}\right)
$$


International Journal of Computer Networks \& Communications (IJCNC) Vol.14, No.1, January 2022

$$
X_{2}^{(i+1)}=X_{2}^{(i)}+\rho\left(W^{(i+1)}-Z^{(i+1)}-U \bar{P}^{(i+1)} V\right)
$$

Because the first subproblem is concerned with optimization over through the variable ${ }^{D}$, now let express by retaining just the words that are relevant to it and rounding the square, i.e.,

$$
D^{(i+1)}=\arg \min _{D} Q_{D}\|D\|_{*}+\rho / 2\left\|D-\left(Z^{(i)}-1 / \rho\left(X_{1}^{(i)}\right)\right)\right\|_{S}^{2}
$$

The answer to eq. (14) is believed to be derived using the Singular Value Thresholding (SVT) function as follows [32]:

$$
D^{(i+1)}=F_{E}^{(i)} \operatorname{diag}\left(\left\{\operatorname{sign}\left(K_{j}^{(i)}\right) \times \max \left(K_{j}^{(i)}, 0\right)\right\}_{1 \leq j \leq r}\right)\left(F_{R}^{(i)}\right)^{G}
$$

where $F_{R}^{(i)}$ and $F_{E}^{(i)}$ contain the right and left singular vectors, of the matrix $\left(Z^{(i)}-1 / \rho\left(X_{1}^{(i)}\right)\right)$, and $K_{j}^{(i)}=\sigma_{j}-Q / \rho$ with ${ }^{r}$ singular values denoted by $\sigma_{j}$. The set of equations must be solved, which necessitates the inversion of the matrix. However, because this matrix is diagonal, the resultant issue is identical to solving the mathematical model shown below;

$$
z=\left(H_{1}+2 \rho I\right)^{-1}\left(X_{1}^{(i)}+\rho D^{(i+1)}+R_{\Omega}+X_{2}^{(i)}+\rho W^{(i)}+\rho H_{2} \bar{P}^{(i)}\right)
$$

Concerning the unidentified variable $\bar{P}$, that may be written correspondingly as; minimization of

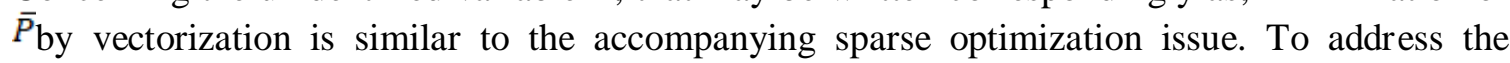
problem, we use the usual LASSO form [33], i.e.

$$
\min _{\bar{P}}\|\bar{P}\|_{1}+\left\|\bar{P}-Y^{(i)}\right\|_{2}^{2}
$$

Where, $Y^{(\bar{i})} \in \mathbb{C}^{E A_{T} A_{R} x 1}$ and a soft-thresholding function may be used to approximate $\bar{p}$ in (17), which is written as follows:

$\bar{p}^{(i+1)}=\operatorname{sign}\left(\operatorname{Real}\left(Y^{(i)}\right)\right)^{\circ} \max \left(\left|\operatorname{Real}\left(Y^{(i)}\right)\right|-Q_{P}^{\prime}, 0\right)+\operatorname{sign}\left(\operatorname{Img}\left(Y^{(i)}\right)\right)^{\circ} \max \left(\left|\operatorname{Img}\left(Y^{(i)}\right)\right|-Q_{P}^{\prime}, 0\right)$

where $Q_{P}^{\prime}=Q P / \rho$ and the sign operators $\operatorname{sign}($.$) and \max ($.$) are used component-wise and it is$ worth noting that the superscript $(i+1)$ will be employed in the suggested evolutionary algorithm. The vector obtained in eq. (18) is then converted into parametric form as $\bar{P}^{(i+1)} \in \mathbb{C}^{E A_{T} \times A_{R}}$.

\section{RESUlts AND ANALYSIS}

In this part, we explore a mmWave $A_{R} \times A_{T}$ MIMO technology for various huge

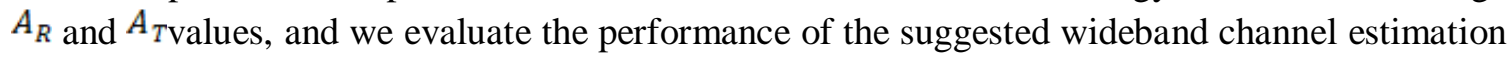
approach for HBF transmission utilising the adaptive random spatial combining design. MATLAB 2016b with system configuration intel i7 processor, 16GB RAM and windows 10 operating system was used to generate all computational simulation results. We particularly simulated the Average Mean Square Error (MSE) performances, which were then averaged across several Monte-Carlo realizations. We also examined the suggested approach's convergence rate and computing expense in comparison to similar state-of-the-art approaches. 
The channel estimation has been conducted in the time domain on receiver across $\mathrm{F}$ frames, using block transmissions with thresholding applied to each sent frame. We expected that the convergence rate of the channels is greater than the period of the F sessions necessary for channel estimation and the learning signal is received by the HBF RX's $A_{R}$ antenna components, which are linked to MR $\beta_{R}<A_{R}$ RF chains. Furthermore, we assumed frequency synchronization and complete time between the transmitting and receiver sides, as well as fully linked proposed systems at all HBF receiver under consideration. We simulated the Normalized Mean-SquareError (NMSE) criterion when evaluating the performance of the proposed algorithm and making comparisons them to relevant state-of-the-art wideband mmWave MIMO channel estimation approaches, that is defined as:

$$
N M S E=\frac{\|\bar{P}-\hat{P}\|}{\|\bar{P}\|}
$$

where $\widehat{\bar{P}}$ symbolises the approximate channel matrix in the beamspace domain. The benchmarking channel estimation approaches under consideration are based on matrix completion and compressive sensing tools. NMSE, is analyzed to avoid bias towards model overestimate / underestimate and giving an impression of the model performance at entire network.

The singular value thresholding (SVT) [32] method is used to solve the rank constrained optimization problem. Although iterative thresholding algorithms, such as Orthogonal Matching Pursuit (OMP) [14], can efficiently solve the L1 minimization with low computational burden, they only deliver excellent estimation accuracy for highly sparse vectors, i.e., when the unknowable vector only has the few non-zero qualities. Message passing techniques, such as Vector Approximate Message Passing (VAMP) [34], on the other hand, give more robust estimate performance in terms of signals with reduced sparsity and also measurements with increasing noise level. These approaches have considered for the comparison with proposed approach.

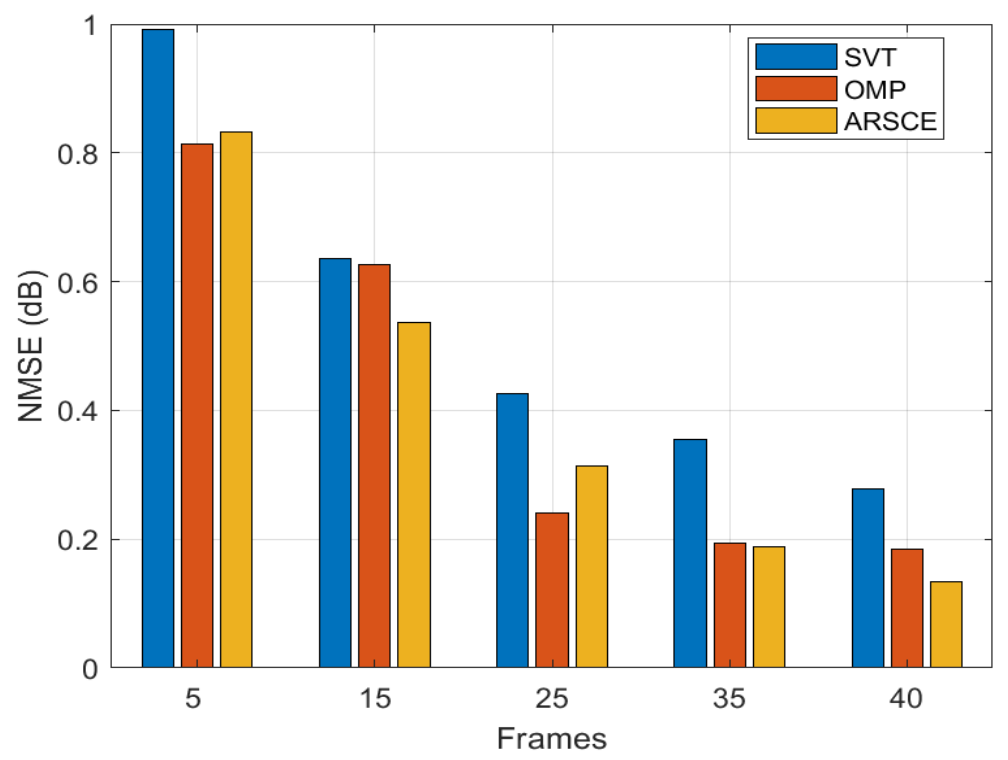

Figure 1. NMSE efficacy as a proportion of training frame number 
International Journal of Computer Networks \& Communications (IJCNC) Vol.14, No.1, January 2022

Considered parameter details for figure 1; 16 number of transmitting antennas, 32 number of the receiving antenna, total number of clusters 4 , channel realization 6,4 receive RF chains, 5 to 40 training frames, and -15 SNR with 20 algorithmic iterations. The NMSE efficacy of the proposed channel estimation approach versus the training frames number $F$ is shown in figure 1, where the training frame numbers; 5, 15, 25, 35 and 40 were considered for the analysis. At training frame 15 , our proposed ARSCE approach has got $15.64 \%$ and $14.25 \%$ less NMSE as compared to SVT and OMP approaches. Similarly at 40 training frames, proposed ARSCE approach has achieved $51.83 \%$ and $27.24 \%$ less NMSE as compared to SVT and OMP approaches. As per the figure 1, it clearly visible that the NMSE values are decrease while increment in training frame numbers and our proposed approach error decreasing w.r.t state-of-the-art methods.

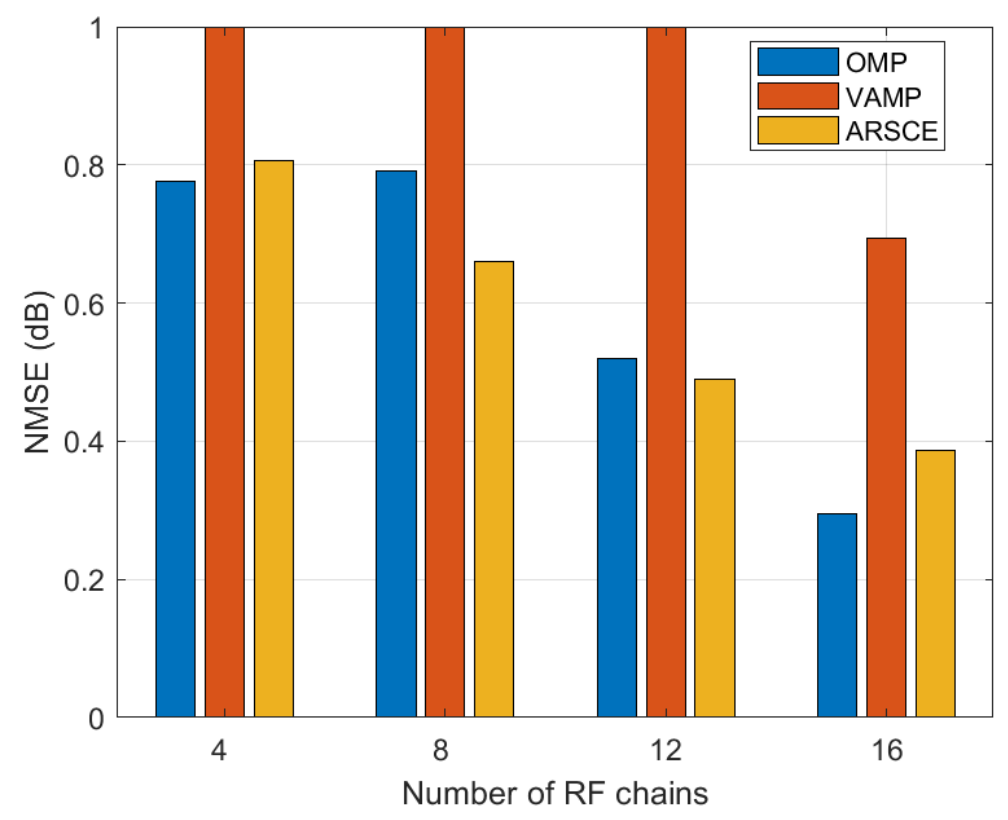

Figure 2. NMSE efficacy as a proportion of RF chains number

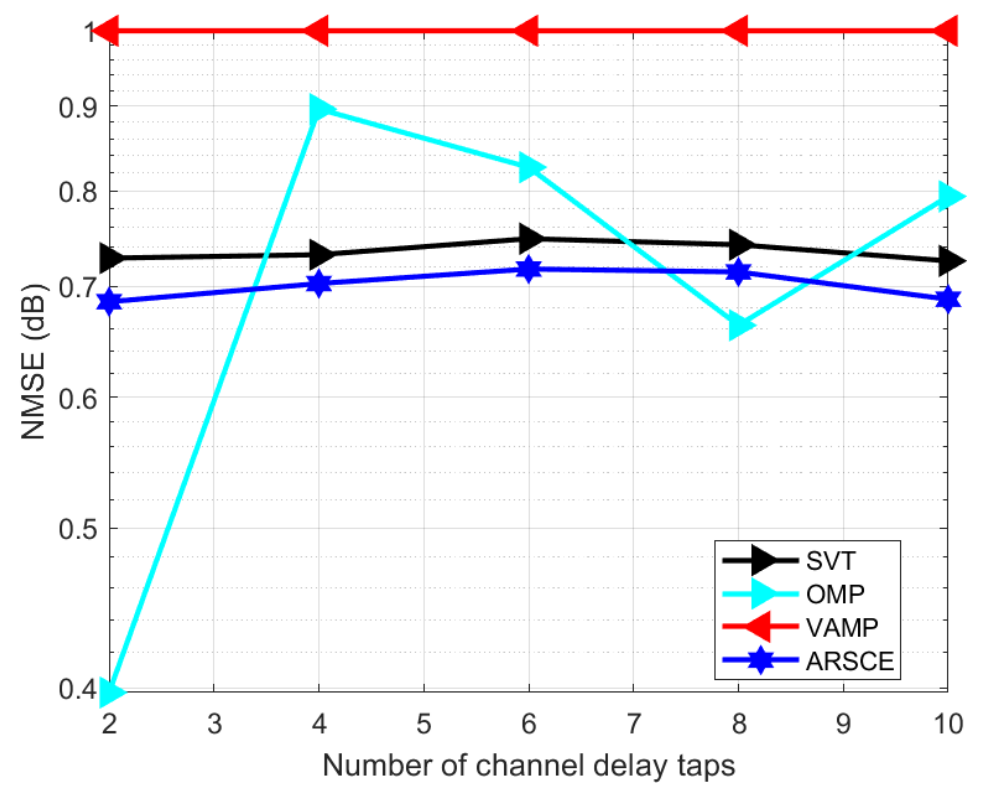

Figure 3. NMSE efficacy as a proportion of channel delay taps number 
International Journal of Computer Networks \& Communications (IJCNC) Vol.14, No.1, January 2022

Considered parameter details for figure 2; 16 number of transmitting antennas, 32 number of the receiving antenna, total number of clusters 4 , channel realization 6,16 receive RF chains, 5 training frames, and -5 SNR with 20 algorithmic iterations. The proposed channel estimation approach NMSE efficacy versus the RF chains number is shown in figure 2, were RF chains number i.e., 4, 8, 12, and 16 considered. At 8 RF chains number, proposed ARSCE approach has achieved $16.63 \%$ and $33.98 \%$ less NMSE as compared to OMP and VAMP approaches. The performance of considered approaches tends to increase while increase in RF chains number. Considered parameter details for figure 3; 16 number of transmitting antennas, 32 number of the receiving antenna, 12 channel propagation paths, total number of clusters 4 , channel realization 6 , 4 receive RF chains, 25 training frames, and -10 SNR with 10 algorithmic iterations.

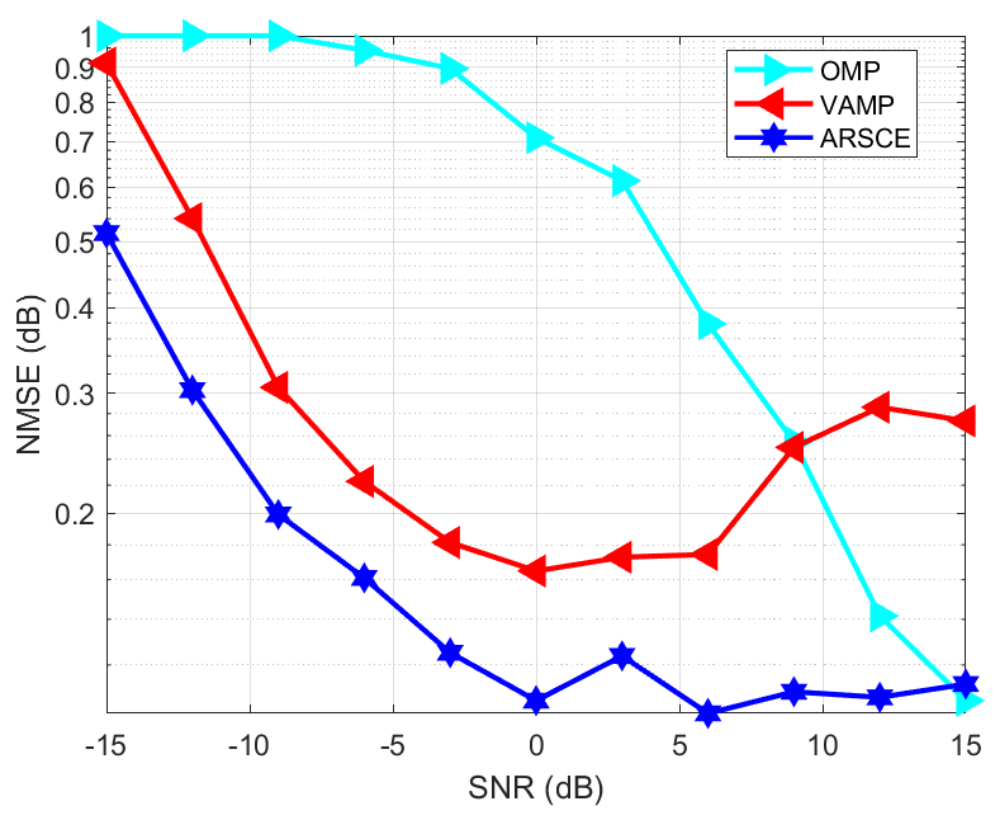

Figure 4. NMSE efficacy as a proportion of SNR

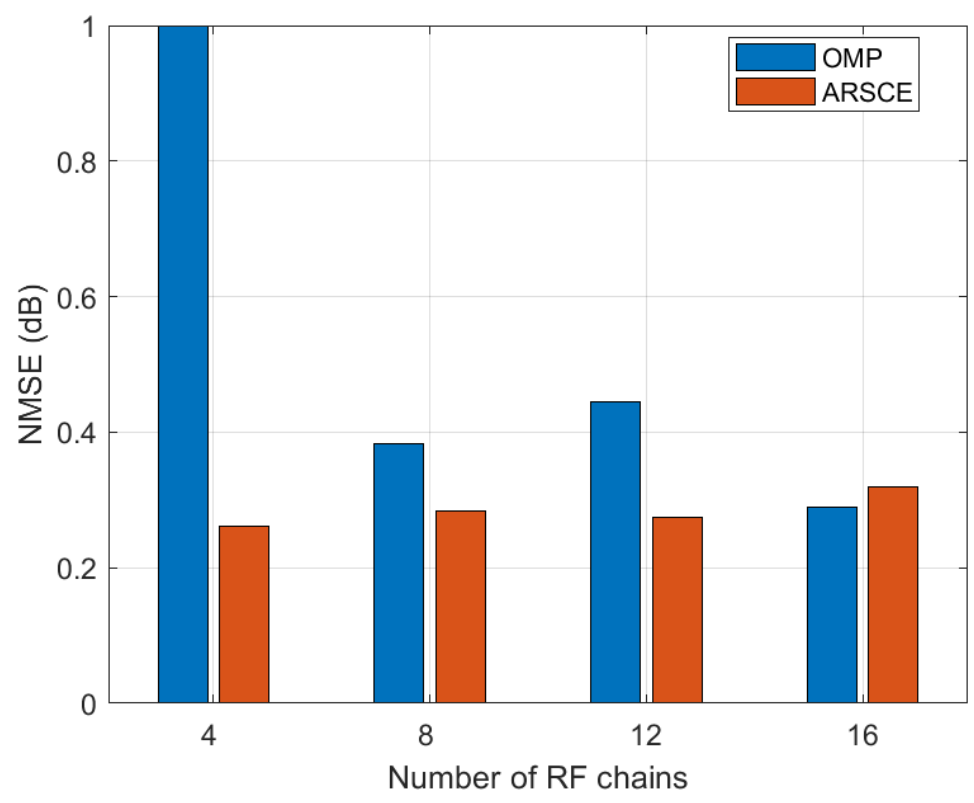

Figure 5. NMSE efficacy as a proportion of RF chains number 
The NMSE efficacy for proposed channel estimation approach versus the channel delay taps number is shown in figure 3, were channel delay taps number i.e., 2, 4, 6, 8, and 10 considered. At 4 channel delay taps number, SVT, OMP and VAMP approaches has 3.93\%, 21.59 and 29.7\% more NMSE as compared to proposed ARSCE approach. The performance is almost stable while changing the channel delay taps number.

At figure 4 the parameter details; 4 number of transmitting antennas, 32 number of the receiving antenna, total number of clusters 4 , channel realization 100, 4 receive RF chains, 35 training frames, and -15 to 15 SNR with 100 algorithmic iterations. Figure 4 shows the NMSE efficacy w.r.t SNR, where SNR varies $-15 \mathrm{~dB}$ to $15 \mathrm{~dB}$ with $3 \mathrm{~dB}$ interval. At $-15 \mathrm{~dB}$ SNR, OMP and VAMP approaches has got $>1 \mathrm{~dB}$ and $0.91 \mathrm{~dB}$ which is $48.57 \%$, and $43.63 \%$ more NMSE as compared to proposed ARSCE approach. Similarly, at 6dB SNR, OMP and VAMP approaches has got $0.37 \mathrm{~dB}$ and $0.17 \mathrm{~dB}$ which is $73 \%$, and $41 \%$ more NMSE as compared to proposed ARSCE approach. The performance is optimized at increasing the SNR.

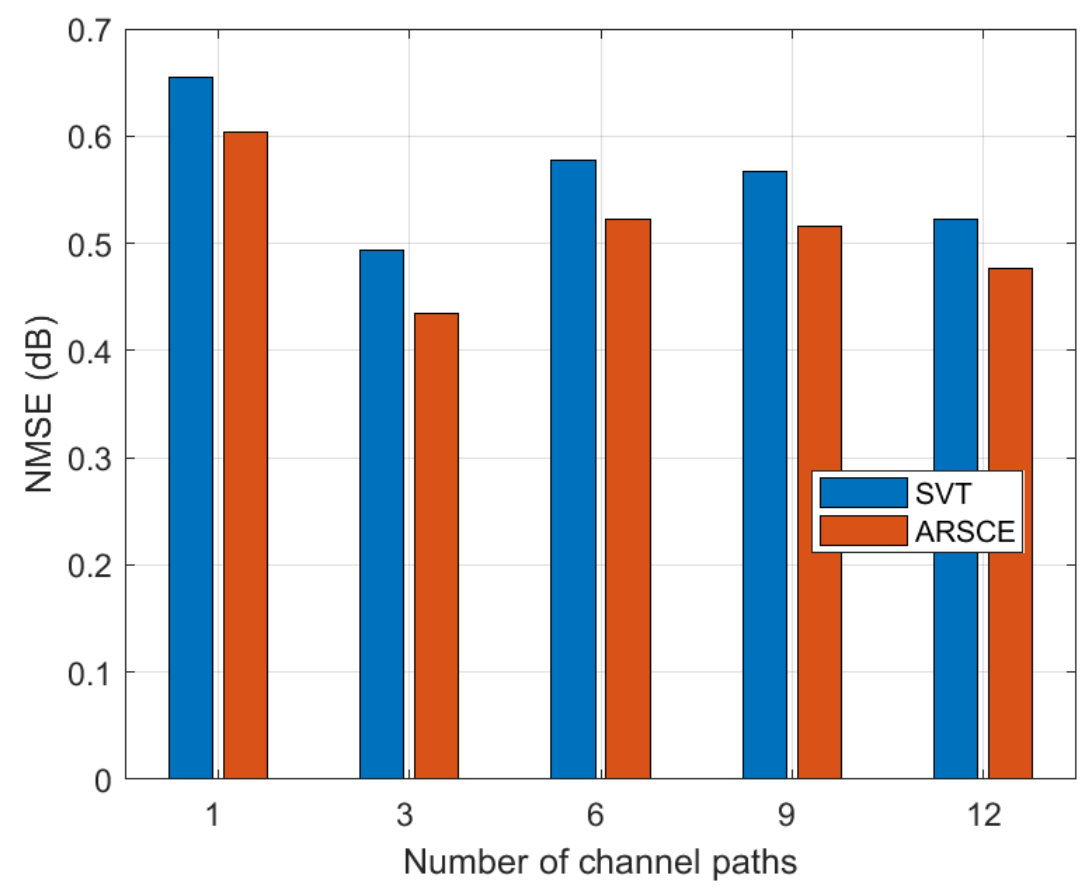

Figure 6. NMSE efficacy as a proportion of channel paths number

Parameter details for figure 5; 4 to16 number of transmitting antennas, 32 number of the receiving antenna, 12 channel propagation paths, total number of clusters 4 , channel realization 6 , 16 receive RF chains, 5 training frames, and $-5 \mathrm{~dB}$ SNR with 20 algorithmic iterations. At figure 5 shows NMSE w.r.t RF chains number, where at 12 RF chains, OMP approach has $38.2 \%$ more NMSE as compared to proposed ARSCE approach. Parameter details for figure 6;16 number of transmitting antennas, 32 number of the receiving antenna, 48 channel propagation paths, total number of clusters 4 , channel realization 1, 4 receive RF chains, 30 training frames, and -5 SNR with 10 algorithmic iterations. Whereas NMSE w.r.t channel paths number has shown in figure 4.6, SVT approach has shown more NMSE as compared to proposed ARSCE approach at various channel paths number. 
International Journal of Computer Networks \& Communications (IJCNC) Vol.14, No.1, January 2022

\section{Conclusions}

In this article, we proposed ARSCE method for wideband mmWave MIMO channel estimates that take use of both the beamspace sparsity and channel's low rank to enable more accurate channel restoration, particularly for short beam learning intervals. The received training signal matrix at the termination of the extended connection is the low-rank matrix sum, that contains the learning signs after passing across the wideband mmWave MIMO system. Extensive simulation findings demonstrate that the suggested methods perform better in terms of MSE for channel estimation when only short beam train lengths are used and while operating in severe noise circumstances. Several approaches shown in above section has considered for the comparison with proposed approach, and proposed ARSCE model has performed well at receiving optimized channel estimation, which result better throughput and better user quality experience.

\section{ACKNOWLEDGEMENTS}

The authors would like to thank everyone, just everyone!

\section{CONFLICTS OF INTEREST}

The authors declare no conflict of interest.

\section{REFERENCES}

[1] F. Boccardi, R. W. Heath Jr., A. Lozano, T. L. Marzetta, and P. Popovski, "Five disruptive technology directions for 5G," IEEE Communication Mag., vol. 52, no. 2, pp. 74-80, Feb. 2014.

[2] T. S. Rappaport, G. R. MacCartney, Jr., M. K. Samimi, and S. Sun, "Wide-band millimeter-wave propagation measurements and channel models for future wireless communication system design," Transactions on Wireless Communications, vol. 63, no. 9, pp. 3029-3056, Sept. 2015.

[3] Y. Niu, Y. Li, D. Jin, L. Su, and A. V. Vasilakos, "A survey of millimeter wave communications (mmWave) for 5G: opportunities and challenges," Wireless Netw., vol. 21, no. 8, pp. 2657-2676, Nov. 2015.

[4] T. S. Rappaport, R. W. Heath Jr., R. C. Daniels, and J. N. Murdock, Millimeter Wave Wireless Communications. Upper Saddle River, NJ: Prentice Hall, 2014.

[5] M. Samimi, K. Wang, Y. Azar, G. N. Wong, R. Mayzus, H. Zhao, J. K. Schulz, S. Sun, F. Gutierrez, Jr., and T. S. Rappaport, " $28 \mathrm{GHz}$ angle of arrival and angle of departure analysis for outdoor cellular communications using steerable beam antennas in New York City," in Proc. IEEE 77th Veh. Technol. Conf. (VTC 2013-Spring), Dresden, Germany, Jun. 2013, pp. 1-6.

[6] R. W. Heath Jr., N. Gonzalez-Prelcic, S. Rangan, W. Roh, and A. M. ' Sayeed, "An overview of signal processing techniques for millimeter wave MIMO systems," IEEE J. Sel. Topics Signal Process., vol. 10, no. 3, pp. 436-453, Apr. 2016.

[7] Y. Azar, G. N. Wong, K. Wang, R. Mayzus, J. K. Schulz, H. Zhao, F. Gutierrez, Jr., D. Hwang, and T. S. Rappaport, "28 GHz propagation measurements for outdoor cellular communications using steerable beam antennas in New York City," in Proc. IEEE Int. Conf. Commun. (ICC), Budapest, Hungary, Jun. 2013, pp. 5143-5147.

[8] L. Lu, G. Y. Li, A. L. Swindlehurst, A. Ashikhmin, and R. Zhang, "An overview of massive MIMO: Benefits and challenges, ” IEEE J. Sel. Topics Signal Processing, vol. 8, no. 5, pp. 742-758, Oct. 2014.

[9] C. B. Peel, B. M. Hochwald, and A. L. Swindlehurst, "A vector-perturbation technique for nearcapacity multiantenna multiuser communication-Part I: Channel inversion and regularization," IEEE Transactions on Wireless Communications, vol. 53, no. 1, pp. 195-202, Jan. 2005.

[10] Q. H. Spencer, A. L. Swindlehurst, and M. Haardt, "Zero-forcing methods for downlink spatial multiplexing in multiuser MIMO channels," IEEE Transactions Signal Process., vol. 52, no. 2, pp. 461-471, Feb. 2004 
International Journal of Computer Networks \& Communications (IJCNC) Vol.14, No.1, January 2022

[11] D. Lee, S.-J. Kim, and G. B. Giannakis, "Channel gain cartography for cognitive radios leveraging low rank and sparsity," IEEE Transactions on Wireless Communications, vol. 16, no. 9, pp. 59535966, Sep. 2017.

[12] X. Li, J. Fang, H. Li, and P. Wang, "Millimeter wave channel estimation via exploiting joint sparse and low-rank structures," IEEE Transactions on Wireless Communications, vol. 17, no. 2, pp. 11231133, Feb. 2018.

[13] E. Vlachos, G. C. Alexandropoulos, and J. Thompson, "Massive MIMO channel estimation for millimeter wave systems via matrix completion," IEEE Signal Process. Lett., vol. 25, no. 11, pp. 1675-1679, Nov. 2018.

[14] K. Venugopal, A. Alkhateeb, N. Gonzalez-Prelcic, and R. W. Heath, Jr., ' "Channel estimation for hybrid architecture-based wideband millimeter wave systems," IEEE J. Sel. Areas Commun., vol. 35, no. 9, pp. 1996-2009, Sep. 2017.

[15] Molisch, A. F., Ratnam, V. V., Han, S., Li, Z., Nguyen, S. L. H., Li, L., \& Haneda, K. (2017). Hybrid beamforming for massive MIMO: A survey. IEEE Communications Magazine, 55(9), 134-141.

[16] J. Du, J. Li, J. He, Y. Guan, \& H. Lin, "Low-complexity joint channel estimation for multi-user mmWave massive MIMO systems.” Electronics, 9, 301. 2020

[17] T. Lin, Y. Zhu, "Beamforming design for large-scale antenna arrays using deep learning. IEEE Wireless Communications Letters, Volume 9, Issue 1, 2020.

[18] Bjornson, E., Larsson, E. G., \& Debbah, M. "Massive MIMO for maximal spectral efficiency: How many users and pilots should be allocated?" IEEE Transactions on Wireless Communications, 15(2), 1293-1308. 2016.

[19] J. Jiang, Y. Li, \& C. Li, "Multitask deep learning-based multiuser hybrid beamforming for mm-wave orthogonal frequency division multiple access systems." Science China Information Sciences, 63, 180303:1-180303:11. 202

[20] Q. Qin, L. Gui, P. Cheng, \& B. Gong, "Time-varying channel estimation for millimeter wave multiuser MIMO systems." IEEE Transactions on Vehicular Technology, 67(10), 9435-9448. 2018.

[21] X. Wu, D. Liu and F. Yin, "Hybrid Beamforming for Multi-User Massive MIMO Systems," in IEEE Transactions on Communications, vol. 66, no. 9, pp. 3879-3891, Sept. 2018.

[22] T. E. Bogale and L. B. Le, "Beamforming for multiuser massive MIMO systems: Digital versus hybrid analog-digital," 2014 IEEE Global Communications Conference, 2014, pp. 4066-4071.

[23] J. Jiang, M. Lei, \& H. Hou, "Downlink multiuser hybrid beamforming for MmWave massive MIMONOMA system with imperfect CSI.” International Journal of Antennas and Propagation, Article ID 9764958, p. 10. 2019.

[24] L. Chen and X. Yuan, "Blind Multiuser Detection in Massive MIMO Channels With Clustered Sparsity," in IEEE Wireless Communications Letters, vol. 8, no. 4, pp. 1052-1055, Aug. 2019, doi: 10.1109/LWC.2019.2906168.

[25] T. Lin, J. Cong, Y. Zhu, J. Zhang and K. Ben Letaief, "Hybrid Beamforming for Millimeter Wave Systems Using the MMSE Criterion," in IEEE Transactions on Communications, vol. 67, no. 5, pp. 3693-3708, May 2019.

[26] Y. Zhang, J. Du, Y. Chen, M. Han and X. Li, "Optimal Hybrid Beamforming Design for MillimeterWave Massive Multi-User MIMO Relay Systems," in IEEE Access, vol. 7, pp. 157212-157225, 2019.

[27] J. Jiang, \& D. Kong "Joint user scheduling and MU-MIMO hybrid beamforming algorithm for mmwave FDMA massive MIMO system." International Journal of Antennas and Propagation, Article ID 4341068, p 10. 2016.

[28] X. Xue, T. E. Bogale, X. Wang, Y. Wang and B. L. Long, "Hybrid analog-digital beamforming for multiuser MIMO millimeter wave relay systems," 2015 IEEE/CIC International Conference on Communications in China (ICCC), 2015, pp. 1-7.

[29] O. E. Ayach, S. Rajagopal, S. Abu-Surra, Z. Pi and R. W. Heath, "Spatially Sparse Precoding in Millimeter Wave MIMO Systems," in IEEE Transactions on Wireless Communications, vol. 13, no. 3, pp. 1499-1513, March 2014.

[30] L. Liang, W. Xu and X. Dong, "Low-Complexity Hybrid Precoding in Massive Multiuser MIMO Systems," in IEEE Wireless Communications Letters, vol. 3, no. 6, pp. 653-656, Dec. 2014.

[31] A. F. Molisch, V. V. Ratnam, S. Han, Z. Li, S. L. H. Nguyen, L. Li, and K. Haneda, "Hybrid beamforming for massive MIMO: A survey," IEEE Communications Mag., vol. 55, no. 9, pp. 134141, Sep. 2017.

[32] J. F. Cai, E. J. Candes, and Z. Shen, "A singular value thresholding algorithm for matrix completion," SIAM J. Opt., vol. 20, no. 4, pp. 1956-1982, 2010. 
International Journal of Computer Networks \& Communications (IJCNC) Vol.14, No.1, January 2022

[33] R. Tibshirani, "Regression shrinkage and selection via the Lasso," J. Royal Stat. Society, Series B, vol. 58, no. 1, pp. 267-288, 1996.

[34] P. Schniter, S. Rangan and A. K. Fletcher, "Vector approximate message passing for the generalized linear model," 2016 50th Asilomar Conference on Signals, Systems and Computers, 2016, pp. 15251529.

\section{AUTHORS}

\section{Rajkumar M Vadgave}

Received the BE in Electronics and Communication Engineering from VTU Belagavi University in 2011 and M.Tech in Communication Systems from VTU Belagavi University in 2013. He is pursuing Ph.D from VTU Belagavi University.He is currently working as Assistant Professor in the Dept of E \& CE Veerappa Nisty Engineering College Shorapur from 2013 to till dated. He has authored or coauthored more than 3 refereed journals and conference papers. His research interest includes wireless sensor network, communication system. He can be contacted at email: rajkumarv074@gmail.com

\section{Manjula S}

Received the B.E in Electronics and Communication Engineering from Gulbarga University in 1997 and M.Tech in Electronics and Communication Engineering from RVD University in 2006 and Ph.D degree from Singhania University in 2014. She became an Assistant professor in 1998, an Associate professor in 2007 and Professor of E $\&$ CE dept in 2014. She has authored or coauthored more than 20 referred journal and conference papers. Her research interest includes Synthesis characterization and microwave properties of conducting polymer composites. Member of ISTE, IETE. She can be contacted at email: manjulasidramshetty@gmail.com

\section{Dr. T.S. Vishwanath}

Ph.D in Control System, Institution: Dr MGR Educational and Research Institute University Chennai India, M. E. in Control System, Institution: WCE Sangli, BE in Electrical Engineering, Institution: PDA College of Engineering Gulbarga. Research work at Robust $\mathrm{H} \infty$ control and analysis" application to electrical drives. Member of Institute of Engneer (MIE), ISTE, IETE and IJEET. He can be contacted at email: tsvrec1@ rediffmail.com
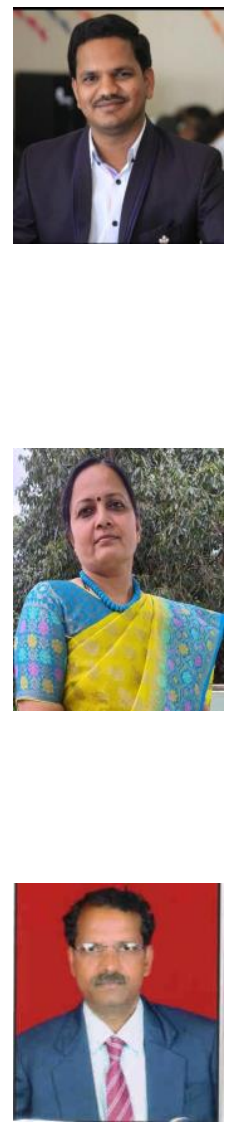